\title{
INTEGRALS INVOLVING $E$-FUNCTIONS AND ASSOCIATED LEGENDRE FUNCTIONS
}

\author{
by T. M. MACROBERT
}

(Received 2nd September, 1954)

$\S 1$. Introductory. The formulae to be proved are as follows.

If $p \geqq q+1, R(l)>0, R\left(\alpha_{r}-l+m+n\right)>-1, R\left(\alpha_{r}-l+m-n\right)>0, r=1,2, \ldots, p$, $\int_{1}^{\infty} E\left\{p ; \alpha_{r}: q ; \rho_{s}: z /(\lambda-1)\right\}(\lambda-1)^{l-1}\left(\lambda^{2}-1\right)^{-\frac{1}{2} m} P_{n}{ }^{-m}(\lambda) d \lambda$

$$
\begin{gathered}
=\frac{\pi}{\Gamma(m+n+1) \Gamma(m-n)} \\
\times\left[\begin{array}{c}
\frac{2^{n} z^{l-m-n-1}}{\cos n \pi \sin (l-m-n) \pi} E\left(\begin{array}{l}
m+n+1, n+1, \alpha_{1}-l+m+n+1, \ldots, \alpha_{p}-l+m+n+1 \quad: \frac{1}{2} e^{ \pm i \pi} \bar{z} \\
2 n+2, m+n-l+2, \rho_{1}-l+m+n+1, \ldots, \rho_{q}-l+m+n+1
\end{array}\right) \\
+\frac{2^{-n-1} z^{l-m+n}}{\cos n \pi \sin (l-m+n) \pi} E\left(\begin{array}{c}
m-n,-n, \alpha_{1}-l+m-n, \ldots, \alpha_{p}-l+m-n: \\
\frac{1}{2} e^{ \pm i \pi} z \\
-2 n, m-n-l+1, \rho_{1}-l+m-n, \ldots, \rho_{q}-l+m-n
\end{array}\right) \\
-\frac{2^{l-m} \sin (l-m) \pi}{\sin (l-m+n) \pi \sin (l-m-n) \pi} E\left(\begin{array}{c}
l, l-m, \alpha_{1}, \ldots, \alpha_{p} \\
l-m+n+1, l-m-n, \rho_{1}, \ldots, \rho_{q}
\end{array}\right.
\end{array}\right]
\end{gathered}
$$

If $p \geqq q+1, R(l+m)>0, R\left(\alpha_{r}-l-m+n\right)>-1, R\left(\alpha_{r}-l-m-n\right)>0, r=1,2, \ldots, p$,

$$
\begin{aligned}
& \int_{1}^{\infty} E\left\{p ; \alpha_{r}: q ; \rho_{s}: z /(\lambda-1)\right\}(\lambda-1)^{l-1}\left(\lambda^{2}-1\right)^{\frac{1}{2} m} P_{n}{ }^{-m}(\lambda) d \lambda \\
& =-\frac{2^{-n-1} \sin (m+n) \pi}{\cos n \pi \sin (l+m+n) \pi} z^{l+m+n} E\left(\begin{array}{l}
-n,-n-m, \alpha_{1}-l-m-n, \ldots, \alpha_{p}-l-m-n \quad: \quad \frac{1}{2} e^{ \pm i \pi} z \\
-2 n, 1-l-m-n, \rho_{1}-l-m-n, \ldots, \rho_{q}-l-m-n
\end{array}\right) \\
& +\frac{2^{n} \sin (m-n) \pi}{\cos n \pi \sin (l+m-n) \pi} z^{l+m-n-1} \\
& \times E\left(\begin{array}{l}
n+1, n-m+1, \alpha_{1}-l-m+n+1, \ldots, \alpha_{p}-l-m+n+1: \quad \frac{1}{2} e^{ \pm i \pi} z \\
2 n+2,2-l-m+n, \rho_{1}-l-m+n+1, \ldots, \rho_{a}-l-m+n+1
\end{array}\right)
\end{aligned}
$$

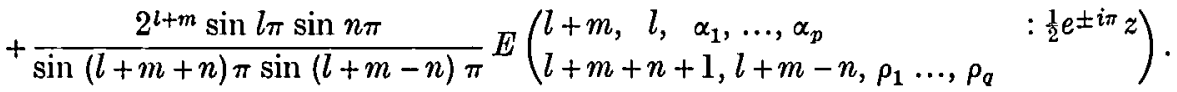

$$
\begin{aligned}
& \text { If } p \geqq q+1, R(l)>0, R(l+m)>0, R\left(\alpha_{r}-l-m+n\right)>-1 \text {, } \\
& \int_{1}^{\infty} E\left\{p ; \alpha_{r}: q ; \rho_{s}: z /(\lambda-1)\right\}(\lambda-1)^{l-1}\left(\lambda^{2}-1\right)^{\frac{1}{m} m} Q_{n}{ }^{-m}(\lambda) d \lambda \\
& =-\frac{\pi 2^{n} z^{l+m-n-1}}{\sin (l+m-n) \pi} E\left(\begin{array}{l}
n+1, n-m+1, \alpha_{1}-l-m+n+1, \ldots, \alpha_{p}-l-m+n+1: \frac{1}{2} e^{ \pm i \pi} z \\
2 n+2,2-l-m+n, \rho_{1}-l-m+n+1, \ldots, \rho_{q}-l-m+n+1
\end{array}\right) \\
& +\frac{\pi 2^{l+m-1}}{\sin (l+m-n) \pi} E\left(\begin{array}{l}
l, \quad l+m, \quad \alpha_{1}, \ldots, \alpha_{p}: \quad \frac{1}{2} e^{ \pm i \pi} z \\
l+m+n+1, l+m-n, \rho_{1}, \ldots, \rho_{q}
\end{array}\right) \text {. }
\end{aligned}
$$

The method of proof is outlined in $\S 2$. Many special cases can be derived from these formulae. An example is given in $\S 3$.

$\S 2$. Proofs of the formulae. The formulae can be deduced from the following three formulae [Q.J.M. XI, 1940, pp. 98, 99].

If $R(z)>0, R(l)>0$, 


$$
\begin{aligned}
\int_{1}^{\infty} E\{:: z /(\lambda-1)\} & (\lambda-1)^{l-1}\left(\lambda^{2}-1\right)^{-\mathrm{l} m} P_{n}{ }^{-m}(\lambda) d \lambda \\
& =\frac{2^{-m} z^{l}}{\Gamma(m+n+1) \Gamma(m-n)} E\left(\begin{array}{c}
m+n+1, m-n, l: 2 / z \\
m+1
\end{array}\right)
\end{aligned}
$$

If $R(z)>0, R(l+m)>0$,

$$
\begin{aligned}
\int_{1}^{\infty} E\{:: z /(\lambda-1)\}(\lambda-1)^{l-1}\left(\lambda^{2}-1\right)^{\frac{1}{2}} P_{n}^{-m}(\lambda) d \lambda \\
=-\frac{\sin n \pi}{\pi} z^{l+m} E\left(\begin{array}{c}
-n, n+1, l+m: 2 / z) \\
m+1
\end{array}\right.
\end{aligned}
$$

If $R(z)>0, R(l+m)>0, R(l)>0$,

$$
\begin{aligned}
\int_{1}^{\infty} E\{:: z /(\lambda-1)\}(\lambda-1)^{l-1}\left(\lambda^{2}-1\right)^{\frac{1}{m}} Q_{n}{ }^{-m}(\lambda) d \lambda \\
\quad=\frac{z^{l}}{2 \sin m \pi}\left\{\begin{array}{l}
\sin n \pi z^{m} E(-n, n+1, l+m: 1+m: 2 / z) \\
-2^{m} \sin (m+n) \pi E(n-m+1,-n-m, l: 1-m: 2 / z)
\end{array}\right\} .
\end{aligned}
$$

On applying the formula, where $p \geqq q+1$,

$$
\begin{aligned}
E\left(p ; \alpha_{r}:\right. & \left.q: \rho_{s}: z\right)=\pi^{p-q-1} \sum_{r=1}^{p}\left\{\prod_{g=1}^{p} \sin \left(\alpha_{s}-\alpha_{r}\right) \pi\right\}^{-1} \prod_{t=1}^{q} \sin \left(\rho_{t}-\alpha_{r}\right) \pi \\
& \times z^{\alpha_{r}} E\left\{\begin{array}{l}
\alpha_{r}, \alpha_{r}-\rho_{1}+1, \ldots, \alpha_{r}-\rho_{q}+1:(-1)^{p-q-1} / z \\
\alpha_{r}-\alpha_{1}+1, \ldots * \ldots, \alpha_{r}-\alpha_{p}+1
\end{array}\right\} \ldots \ldots \ldots \ldots
\end{aligned}
$$

to the R.H.S.'s of (4), (5) and (6), formulae (1), (2), (3), with $p=q=0$ are obtained. The formulae can then be generalised in the usual way.

§ 3. Integral involving a Product of two Associated Legendre Functions. In (3) take $z=2, p=2, q=1, \alpha_{1}=q-p, \alpha_{2}=q+p+1, \rho_{1}=q+1$, apply the formula

$$
E\{q-p, q+p+1: q+1: 2 /(\lambda-1)\}=2^{q} \Gamma(q-p) \Gamma(q+p+1)\left(\lambda^{2}-1\right)^{-1} q P_{p}^{-q}(\lambda),
$$

and so obtain the following result.

If $R(l)>0, R(l+m)>0, R(q+p-m+n-l)>-2, R(q-p-m+n-l)>-1, R(m-q)>-1$

$$
\begin{aligned}
& \int_{1}^{\infty}(\lambda-1)^{l-1}\left(\lambda^{2}-1\right)^{-\frac{1}{d} q} P_{p}^{-q}(\lambda)\left(\lambda^{2}-1\right)^{\frac{1}{3} m} Q_{n}{ }^{-m}(\lambda) d \lambda \\
& \quad=\frac{\pi 2^{l+m-q-1}}{\Gamma(q-p) \Gamma(q+p+1) \sin (l+m-n) \pi} \\
& \times\left[\begin{array}{l}
E\left(\begin{array}{l}
l, l+m, q-p, q+p+1: e^{ \pm i \pi} \\
l+m+n+1, l+m-n, q+1
\end{array}\right) \\
-E\left(\begin{array}{l}
n+1, n-m+1, q-p-l-m+n+1, q+p-l-m+n+2: e^{ \pm i \pi} \\
2 n+2,2-l-m+n, q-l-m+n+2
\end{array}\right)
\end{array}\right]
\end{aligned}
$$

\section{The University}

\section{Glasgow}

